\title{
Decay kinetics of HIV-1 specific T cell responses in vertically HIV-1 exposed seronegative infants
}

\author{
Sara J. Holditch ${ }^{1}$, Emily M. Eriksson ${ }^{1}$, Leandro F. Tarosso ${ }^{2}$, Peter J. Kuebler ${ }^{1}$, Esper G. Kallas ${ }^{2}$, \\ Erik K. Nielsen ${ }^{3}$, Andrew A. Wiznia ${ }^{3}$, Michael G. Rosenberg ${ }^{3}$ and Douglas F. Nixon ${ }^{1}$ *
}

Division of Experimental Medicine, Department of Medicine, San Francisco General Hospital, University of California San Francisco, San Francisco, CA, USA

${ }^{2}$ Division of Clinical Immunology and Allergy, School of Medicine, University of São Paulo, São Paulo, Brazil

3 Jacobi Medical Center, Albert Einstein College of Medicine, Bronx, NY, USA

\section{Edited by:}

Sharon Lewin, Alfred Health and Monash University, Australia

Reviewed by:

Paul Klenerman, University of Oxford, UK

Michael Betts, University of

Pennsylvania, USA

*Correspondence:

Douglas F. Nixon, Department of

Experimental Medicine, Building 3,

Room 601, 1001 Potrero Avenue, San

Francisco, CA 94110, USA.

e-mail:douglas.nixon@ucsf.edu
Objective: The majority of infants born, in developed countries, to HIV-1 positive women are exposed to the HIV-1 virus in utero or peri/post-partum, but are born uninfected. We, and others, have previously shown HIV-1 specific T cell responses in HIV-1 exposed seronegative (HESN) neonates/infants. Our objective in this study was to examine the rate of decay in their HIV-1 specific T cell response over time from birth. Design: Cross-sectional and longitudinal studies of HIV-1 specific T cell responses in HESN infants were performed. Methods: Peripheral blood mononuclear cells (PBMC) were isolated from 18 HIV-1 DNA PCR negative infants born to HIV-1 infected mothers receiving care at the Jacobi Medical Center, Bronx, NY, USA. PBMC were examined for T cell responses to HIV-1 antigens by interferon-gamma (IFN- $\gamma$ ) ELISPOT. Results: PBMC from 15 HESN neonates/infants were analyzed. We observed a decay of HIV-1 specific T cell responses from birth at a rate of -0.599 spot forming unit $/ 10^{6}$ cells per day, with a median half-life decay rate of 21.38 weeks (13.39-115.8). Conclusion: Our results support the dynamic nature of T cell immunity in the context of a developing immune system. The disparate rate of decay with studies of adults placed on antiretroviral drugs suggests that antigen specific $T$ cell responses are driven by the natural rate of decay of the T cell sub-populations themselves.

Keywords: HIV-1, exposed seronegative, vertically exposed, neonates, T cells

\section{INTRODUCTION}

Several reports, including from our group, have identified HIV-1 specific T cell responses in HIV-1 Exposed Seronegative (HESN) infants born to HIV-1 infected mothers (Cheynier et al., 1992; Rowland-Jones et al., 1993; Aldhous et al., 1994; De Maria et al., 1994; Kuhn et al., 2001; Legrand et al., 2006). The role of these T cell responses in helping to prevent infection is unknown. However, reports of significant anti-viral immune responses against congenital pathogens in utero (Rowland-Jones et al., 1993; Kuhn et al., 2001; Marchant et al., 2003; Legrand et al., 2006; Miles et al., 2008; Mold et al., 2008; John-Stewart et al., 2009) suggest anti-HIV1 specific $\mathrm{T}$ cell responses may in part contribute to protection against vertical transmission.

Maternal viral suppression with antiretroviral agents, together with close intrapartum and post-partum monitoring of viral loads, is the standard of care. Despite efforts to control maternal viral load, pregnant, or breast-feeding mothers, may experience fluctuations in viremia (Van Sighem et al., 2008). These periods of variable viral load pose an increased risk of exposure to and vertical transmission of HIV-1 during pregnancy, peripartum, and post-partum (Rowland-Jones et al., 1993; Kuhn et al., 2001; Sabbaj et al., 2002, 2005; Sharp et al., 2005; John-Stewart et al., 2009; Walter et al., 2009). Uninfected infants born to HIV-1 infected mothers (HESN infants) could provide valuable insight into the kinetics of HIV-1 specific $\mathrm{T}$ cell responses in exposed uninfected individuals.

\section{MATERIALS AND METHODS}

\section{SUBJECTS}

A cohort of 18 HESN infants born to HIV-1 infected mothers receiving medical care through the Jacobi Medical Center (Bronx, NY, USA) were selected to study the decay kinetics of HIV-1 specific $\mathrm{T}$ cell responses.

\section{BLOOD SAMPLES FROM HESN INFANTS}

EDTA whole blood samples $(2-5 \mathrm{ml})$ from HESN infants were collected during scheduled visits after obtaining written parental/guardian informed consent, according to local institutional review board-approved protocols. Peripheral blood mononuclear cells (PBMCs) from donors were isolated by FicollPaque PLUS density gradient centrifugation (Amersham Pharmacia Biotech, Uppsala, Sweden) and cryopreserved, as previously described (Sharp et al., 2005; Ballan et al., 2007). Healthy, HIV-1 unexposed infant PBMC were unavailable for comparison.

\section{HEALTHY BLOOD DONOR CONTROLS}

Healthy blood donor (HBD) controls were collected from the Stanford Blood Bank as Buffy Coats, isolated by Ficoll-Paque PLUS density gradient centrifugation (Amersham Pharmacia Biotech, Uppsala, Sweden) and cryopreserved, as previously described (Sharp et al., 2005; Ballan et al., 2007). 


\section{ELISPOT ASSAY}

HIV-1 peptide pools were used at $5 \mu \mathrm{g} / \mathrm{ml}$ while Phytohemagglutinin (PHA $2 \mu \mathrm{g} / \mathrm{ml}$; Sigma, Aldrich, UK) and a CMV, influenza, and EBV peptide pool (CEF; NIH AIDS reagent program) were used as positive controls. Unstimulated cells incubated in RPMI1640 with $10 \%$ FBS (R-10) served as the negative control. About 100,000 PBMCs, per well were incubated with all antigens and tested in duplicate wells. Spot forming units (SFUs) were counted with an automated ELISPOT reader (AID-GmbH, Strasberg, Germany). Positive responses were predefined as the mean SFU above zero after subtraction of twice the response to R-10. Negative control wells were required to have less than 20 Spots/well/ $1 \times 10^{5}$ PBMCs. Three HESN infants were removed from analysis due to exceeding negative control limits and negative responses to positive controls.

\section{STATISTICAL METHODS}

Samples were plated in duplicate wells, with the average of well responses used in all calculations. Statistical analyses were performed using Prism 4.0 (GraphPad Software, Inc., CA, USA). Statistical tests used in analysis included Spearman non-parametric correlations, linear, and non-linear regressions (one phase exponential decay), one-way ANOVA's using Kruskal-Wallis, Chisquared tests, Fischer's exact tests, and Dunn's comparison tests.
To address outliers, all statistical tests were run twice; once with the outlier HESN infant, and once with the infant removed. Results reported represent the data set including the outlier HESN infant unless otherwise stated.

\section{RESULTS}

\section{DETERMINATION OF LACK OF HIV-1 INFECTION IN HESN INFANTS}

We screened PBMC from 18 HIV-1 DNA PCR negative HESN infants aged 0-60 months for HIV-1 specific $\mathrm{T}$ cell responses. HIV-1 infected pregnant women and their newborns were managed according to established pMTCT guidelines, including seasonal vaccination against influenza. HIV-1 infected mothers on HAART were given intrapartum IV Zidovudine (ZDV) and their offspring received 6 weeks of oral ZDV postnatally. No infant in this study was breast-fed as per prevailing guidelines during the sample acquisition period. Qualitative HIV-1 DNA PCRs were performed at birth and again at $0.5,1,2$, and 4 months after birth to determine infection status. HIV-1 serologies were performed at approximately 15-24 months of age to confirm the loss of passively acquired HIV-1 specific maternal antibodies.

\section{T CELL RESPONSES IN HESN}

Study participants were screened for interferon-gamma (IFN- $\gamma$ ) responses in an ELISPOT assay against nine HIV-1 antigen peptide pools comprised of 15 mers with an 11aa overlap, acquired

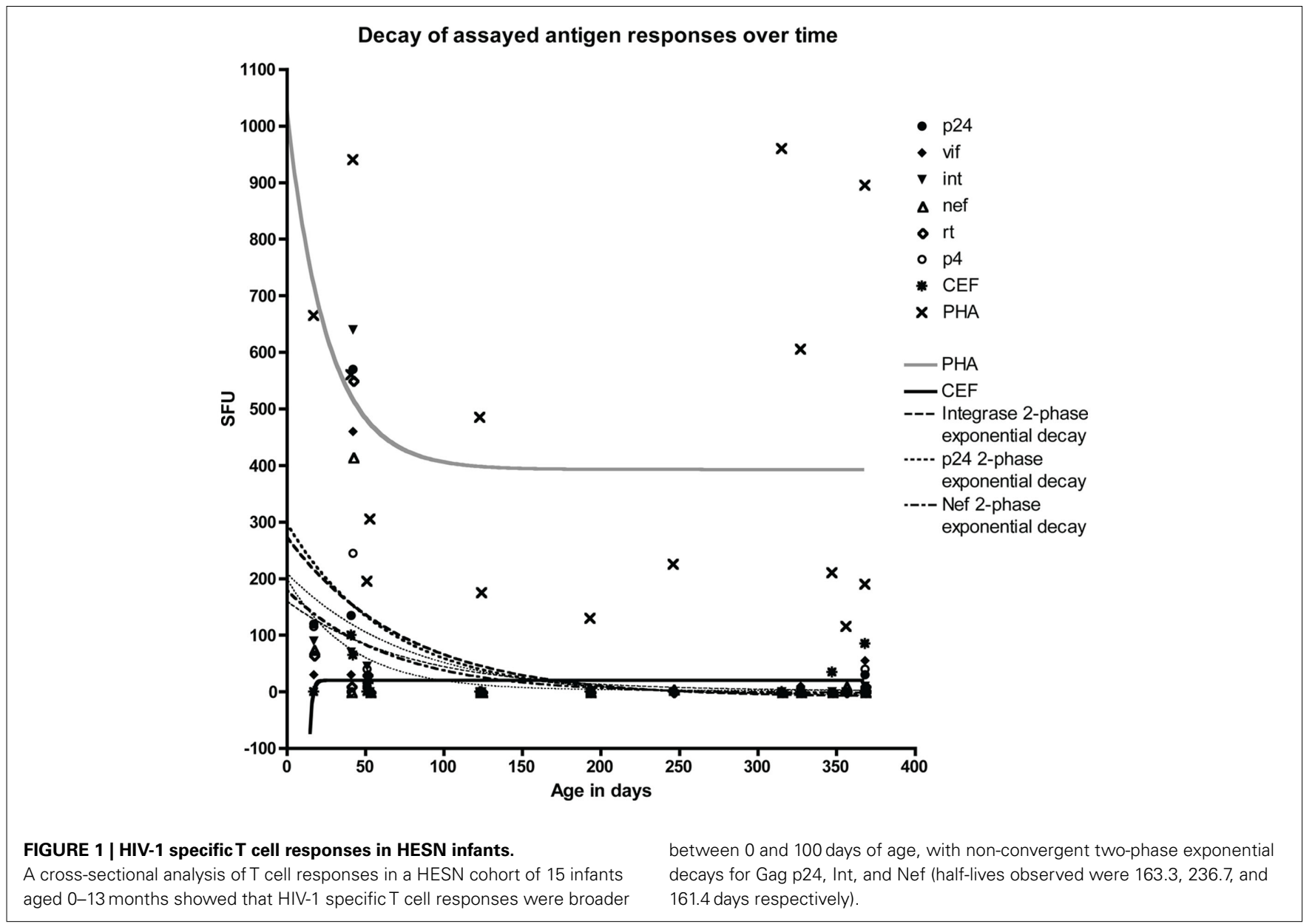


from the NIH AIDS reagent program. PBMC were tested in the ELISPOT assay with peptide pools from HIV-1 consensus B Gag p24 (58 peptides), Nef (48 peptides), Vif (46 peptides), integrase/Int (67 peptides), reverse transcriptase/RT (140 peptides), and protease (24 peptides; NIH AIDS Reagent Program). For interassay variability and plating variability, HBD were plated on each plate per assay day, and analyzed for variation and HIV-1 peptide pool sensitivity. Decay rates, and the range of responses observed in HESN infants were unaffected by interassay variability or HBD HIV-1 specific peptide responses. A crosssectional analysis of $\mathrm{T}$ cell responses of 18 HESN infants aged 0-12.3 months revealed 15 of 18 assayed HESN infants had positive PHA responses (making 15 eligible for analysis). Four of 15 HESN infants had positive CEF responses (coinciding with few EBV, influenza, and CMV seropositive infants; Figure 1). HIV1 specific $\mathrm{T}$ cell responses were predominantly found within the first 100 days of birth. A single response was observed at day 368 . This response was handled as an outlier due to its lack of temporal proximity to other responses (Figure 1). The majority of
HESN infants responded to one or more HIV-1 antigen pools. The mean HIV-1 specific antigen response for infants, aged 0100 days was $126 \times 10^{6} \mathrm{SFU}(0-640 \mathrm{SFU})$, and the mean number of HIV-1 antigen pools responded to by these infants was 4.4 $(0-7)$. There was variable responsiveness to PHA. Monophasic exponential decay analysis of the data found HIV-1 peptide pools of Gag p24, Vif, Integrase, Nef, RT, and protease had half-lives of $44.65,53.10,52.31,45.82,53.27$, and 23.26 days respectively (Mean HIV-1 antigen half-life was 46.24 days; Figure 1). Statistically significant differences were observed in mean antigen responses between two groups of infants, those aged $0-100(n=5)$ and 201-368 $(n=7)$ days from birth, for Gag p24 $(p<0.05)$, RT $(p<0.05)$, and Integrase $(p<0.05)$ peptide pools (Figure $2 \mathbf{B}$ ), with decreases in IFN- $\gamma$ responses after 100 days. Differences between HIV-1 peptide pool responses between infants under, and infants over 100 days of age were found significant $(p=0.039$, Chi-square; Figure 2A). There were trends toward differences between decreasing age and responses for the other HIV-1 antigens measured.

\section{A}

\section{Comparing HESN responders by Age}

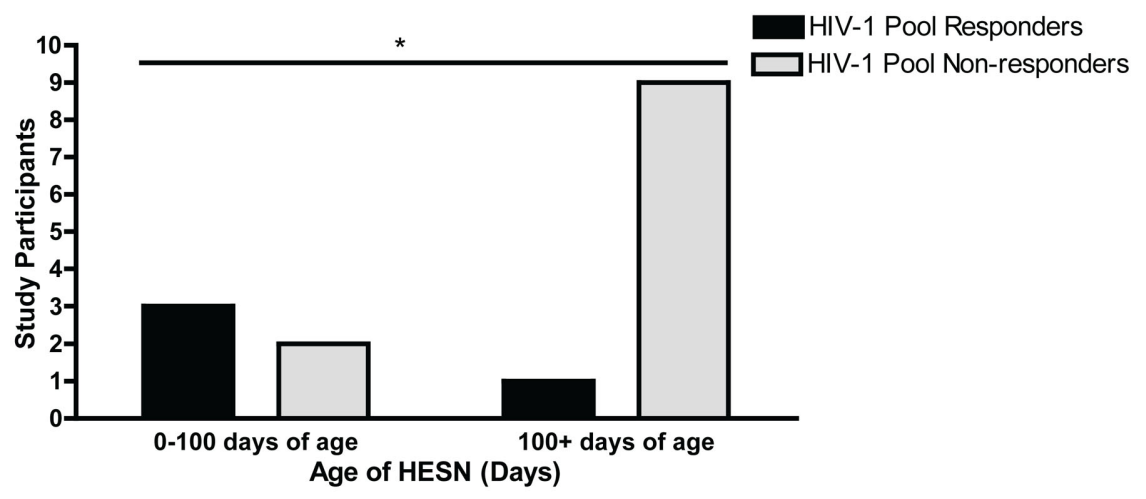

B

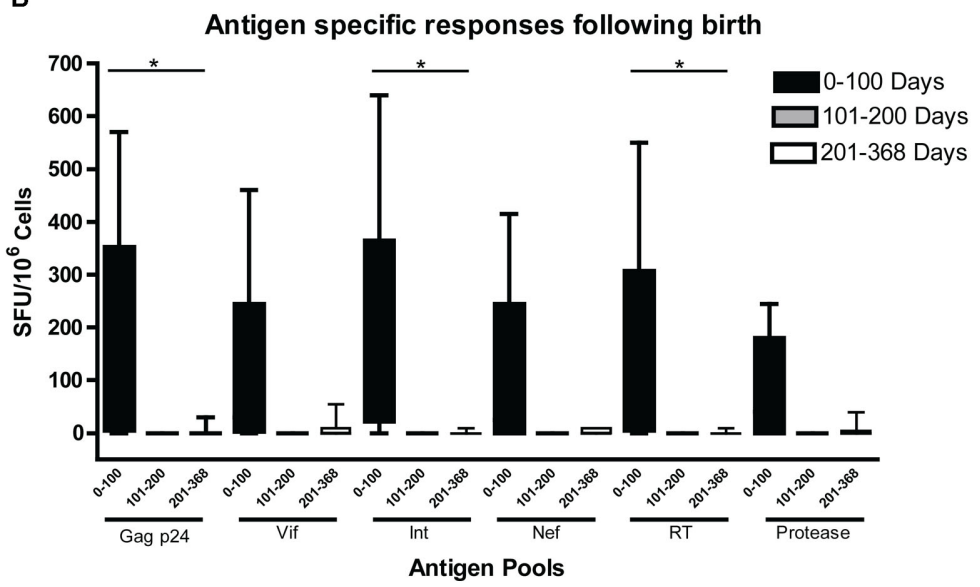

FIGURE 2 | Differences between HIV-1 peptide pool responses between infants under, and infants over 100 days of age were found significant ( $\boldsymbol{p}=\mathbf{0 . 0 3 9}$, Chi-square) (A). Responses were predominantly found within the first 100 days from birth, with Statistically significant differences observed in mean antigen responses between two groups of infants, those aged 0-100 $(n=5)$ and 201-368 $(n=7)$ days from birth, for Gag p24 ( $p<0.05)$, RT $(p<0.05)$, and Integrase $(p<0.05)$ peptide pools (B). 
A

Infant A

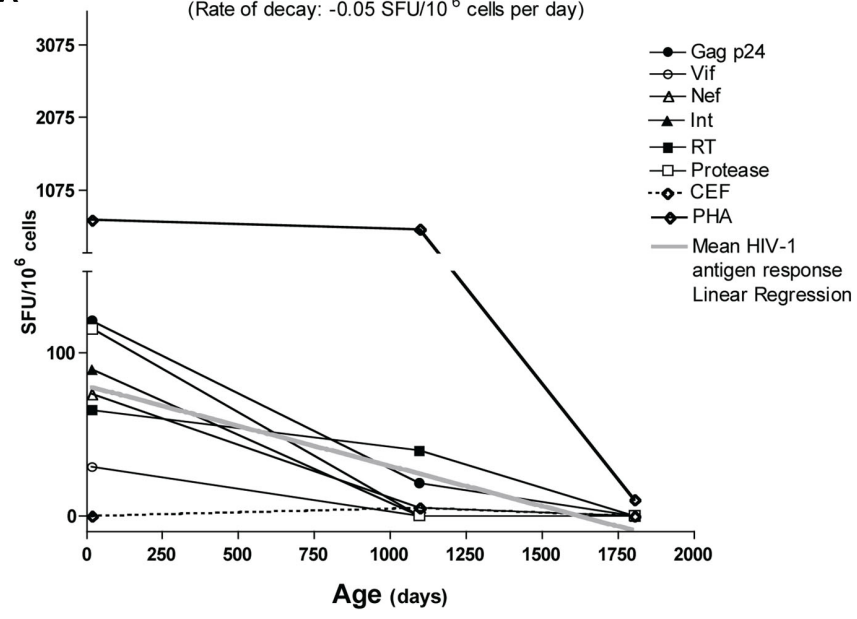

B

Infant B

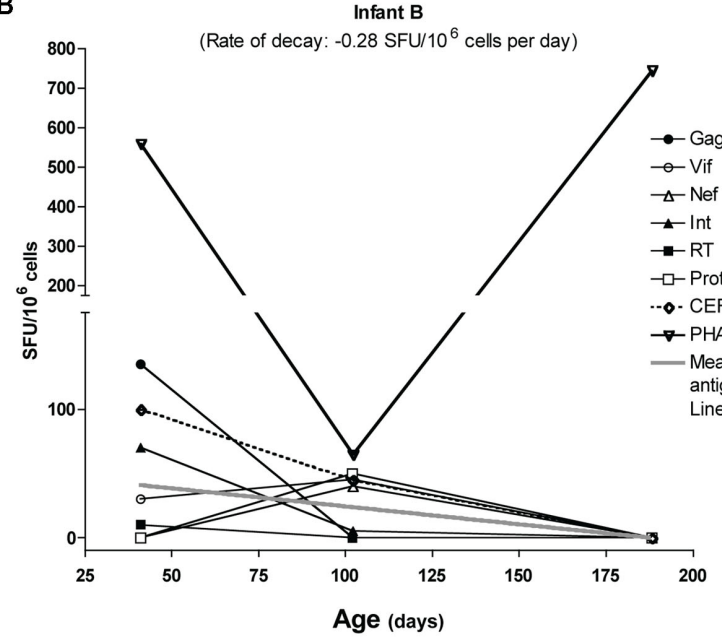

C

Infant C
${ }^{800} \quad$ (Rate of decay: -1.47 SFU $/ 10^{6}$ cells per day)

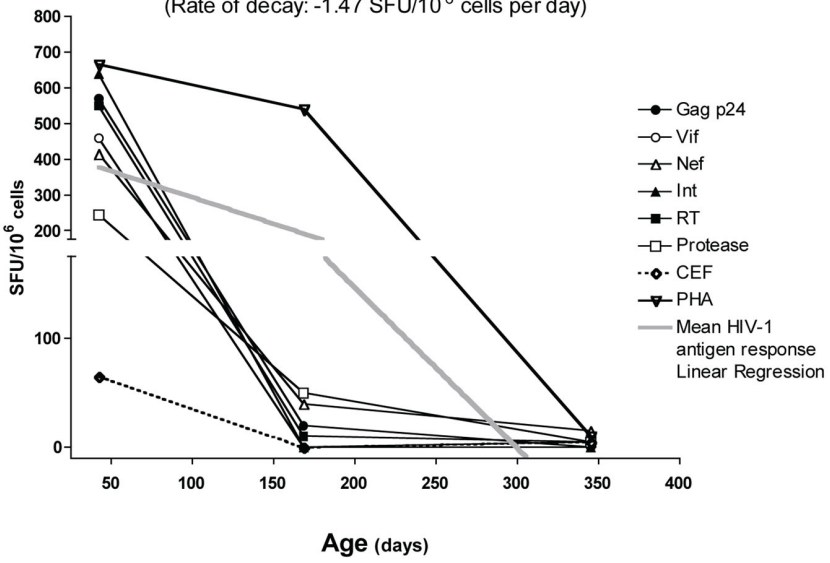

FIGURE 3 | Longitudinal analysis of three infants aged 0-60 months showed no significant differences between the slope-derived decay rates per HIV-1 specific antigen pool response, nor significant differences in

rates of decay between infants $(\mathbf{A}-\mathbf{C})$. From this, we calculated the mean antigen response decay rate of $-0.599 \mathrm{SFU} / 10^{6}$ cells per day, with a median half-life decay rate of 21.38 weeks (13.39-115.8).

Interferon-gamma responses decayed rapidly after day 100 with low mean antigen responses both from days 101-200 of 0 SFU and days 201-368 of $4.41(0-55)$ SFU (Mean antigen responses for days 201-368 were 0.83 (0-10) with the outlier HESN removed). There 
was a decline in the mean number of pools responded to, with 0.0 and 1.43 pools (0-6) for days 101-200 and 201-368, respectively (Pool responses drop to 0.57 for days 201-368 with Outlier removed; Figure 1).

A longitudinal study of three HESN infants with available PBMC time points was conducted to obtain a more in-depth analysis of HIV-1 specific T cell response decay kinetics. An estimated rate of decay (SFU/day) was calculated from the slopes of individual antigen responses per infant. No significant differences were observed in the decay rates per antigen between the infants studied or in the intercepts of specific antigens. These data allowed us to calculate a mean HIV-1 antigen decay rate for all three infants of $-0.599 \mathrm{SFU} /$ day). From this, the half-life of antigen responses was calculated as 21.38 weeks (115.8-13.39; Figures 3A-C).

\section{DISCUSSION}

We found that HIV-1 specific antigen responses were stronger and broader in HESN neonates/infants at the time nearest to birth, and decayed both in magnitude and breadth as time from birth progressed. This observation reinforces previous studies showing that neonates can mount a vigorous virus-specific response at the time of birth or shortly thereafter. Although we contend that the decay in the magnitude and breadth of HIV-1 antigen specific immune response in these HESN infants is due to the absence of viral antigens, we cannot rule out an effect from postpartum ZDV exposure, or the emergence of a regulatory immune cell population, which might suppress HIV-1 specific immune responses.

Previous studies have measured decay rates for HIV-1 and CMV specific epitopes in eight treatment naïve HIV-1 infected adults initiating HAART (Casazza et al., 2001). In that study, a rapid decline was observed in HIV-1 specific CD8+ T cell responses at HAART initiation, with a subsequent rebound in HIV-1 specific CD8+ $\mathrm{T}$ cell responses during treatment interruptions. The authors concluded that the decrease in HIV-1 CD8 + T cell responses represented a normal memory response to the withdrawal of HIV-1 antigen by HAART and calculated a median half-life rate of decay of 38 weeks (Casazza et al., 2001). This was much longer than the median decay rate of 45 days previously measured in a cohort of treated HIV-1 infected adults (Ogg et al., 1999), a value that may specifically reflect an early, rapid phase of decay of $\mathrm{T}$ cell responses after HAART initiation. This might be followed by a much slower decay rate, which would markedly influence the calculation of the aggregate or overall decay rate. The kinetics of decay observed in HESN infants is not similar to that in adults, which may be accounted for by the presence of replicating virus in adult cohorts, where virus replication cannot be demonstrated in these uninfected children. Previous studies with breast-feeding permitted in prenatal care guidelines, have documented robust HIV-1 specific CD4+ T helper responses in HIV-1 exposed uninfected newborns. Such T helper responses could extend the activity of IFN- $\gamma$ secreting HIV-1 specific CTLs, which in turn may protect against post-partum infection.

The ability of HESN infants to mount a memory mucosal immune response was not addressed in our study due to the prohibiting of breast-feeding. Future studies of breast-fed HESN infants could address the persistence of memory CTL responses in the absence of viral replication or antigen presentation. Such data could further address differences in the immune responses between adults and children. It is interesting to observe measurable HIV-1 specific T cell responses in infants whom are likely to have had minimal exposure to HIV-1 antigen (the majority of mothers were on ARV treatment), but it also points to the possibility of a low dose virus stimulus for a higher HIV-1 specific $\mathrm{T}$ cell response. We have used the standardized nomenclature of HESN, but we do not know the true viral burden that these infants were exposed to. It would have been desirable to study the decay of responses to other antigens, and to study an unexposed infant cohort, but due to limited cell numbers, and ethical difficulties in obtaining serial samples from healthy infants, these studies were not performed. We also cannot definitely establish whether the $\mathrm{T}$ cells have redistributed out of the blood, or have the capacity to be reactivated. Another reason for the apparent decline could be simply a change in functionality from interferon $-\gamma$ production to a resting population with more proliferative capacity and less immediate effector function.

In summary, our results support previous reports of HIV-1 specific T cell responses in HESN infants and the dynamic nature of $\mathrm{T}$ cell immunity in the context of a developing immune system. If HIV-1 specific $\mathrm{T}$ cell responses are contributing to protection against infection in neonates, our data supports a therapeutic vaccine approach to boost responses over the time an infant would be breast-fed. Our calculated rate of decay in these HESN infants was not comparable to that measured in adults starting antiretroviral therapy, suggesting that the natural rate of decay of antigen specific T cell sub-populations may be a significant contributor to the overall kinetics of antigen responses in addition to continued antigenic exposure.

\section{ACKNOWLEDGMENTS}

The CEF pool and HIV-1 peptides were obtained through the AIDS Research and Reference Reagent Program, Division of AIDS, NIAID, NIH: (CEF, Gag p24, Nef, Vif, integrase, protease, and reverse transcriptase). Sara J. Holditch, Emily M. Eriksson, and Douglas F. Nixon designed the research; Michael G. Rosenberg, Erik K. Nielsen, and Andrew A. Wiznia provided HESN infant specimens and analyzed data. Sara J. Holditch and Leandro F. Tarosso conducted the cross-sectional laboratory research; Sara J. Holditch conducted the longitudinal laboratory research; Sara J. Holditch and Leandro F. Tarosso analyzed the data; Sara J. Holditch and Douglas F. Nixon prepared the manuscript. Sara J. Holditch, Emily M. Eriksson, Leandro F. Tarosso, Peter J. Kuebler, Esper G. Kallas, Andrew A. Wiznia, Michael G. Rosenberg, and Douglas F. Nixon contributed to the composition of, and proofed, the manuscript. The authors would like to thank Dr. Steve Shiboski, from CTSI for help with statistical analysis, members of the Nixon lab for assistance in processing blood samples, and Janet Young of NIAID for helpful discussions. Funding: This work was supported by grants from the NIH: AI87131, AI60379, NIH/NCRR UCSF-CTSI Grant Number UL1 RR024131, Fundação de Amparo a Pesquisa do Estado de São Paulo (04/15856-9/Kallas and 2010/05845$0 /$ Kallas and Nixon), LFT had a scholarship supported by the Conselho Nacional de Desenvolvimento Científico e Tecnológico (CNPq), Brazilian Ministry of Science and Technology. 


\section{REFERENCES}

Aldhous, M. C., Watret, K. C., Mok, J. Y., Bird, A. G., and Froebel, K. S. (1994). Cytotoxic T lymphocyte activity and CD8 subpopulations in children at risk of HIV infection. Clin. Exp. Immunol. 97, 61-67.

Ballan, W. M., Vu, B. A., Long, B. R., Loo, C. P., Michaelsson, J., Barbour, J. D., Lanier, L. L., Wiznia, A. A., Abadi, J., Fennelly, G. J., Rosenberg, M. G., and Nixon, D. F. (2007). Natural killer cells in perinatally HIV-1-infected children exhibit less degranulation compared to HIV-1-exposed uninfected children and their expression of KIR2DL3, NKG2C, and NKp46 correlates with disease severity. $J$. Immunol. 179, 3362-3370.

Casazza, J. P., Betts, M. R., Picker, L. J., and Koup, R. A. (2001). Decay kinetics of human immunodeficiency virus-specific CD8 $+\mathrm{T}$ cells in peripheral blood after initiation of highly active antiretroviral therapy. J. Virol. 75, 6508-6516.

Cheynier, R., Langlade-Demoyen, P., Marescot, M. R., Blanche, S., Blondin, G., Wain-Hobson, S., Griscelli, C., Vilmer, E., and Plata, F. (1992). Cytotoxic T lymphocyte responses in the peripheral blood of children born to human immunodeficiency virus-1-infected mothers. Eur. J. Immunol. 22, 2211-2217.

De Maria, A., Cirillo, C., and Moretta, L. (1994). Occurrence of human immunodeficiency virus type 1 (HIV-1)-specific cytolytic $\mathrm{T}$ cell activity in apparently uninfected children born to HIV-1infected mothers. J. Infect. Dis. 170, 1296-1299.

John-Stewart, G. C., Mbori-Ngacha, D., Payne, B. L., Farquhar, C., Richardson, B. A., Emery, S., Otieno, P., Obimbo, E., Dong, T., Slyker, J., Nduati, R., Overbaugh, J., and
Rowland-Jones, S. (2009). HV-1specific cytotoxic $\mathrm{T}$ lymphocytes and breast milk HIV-1 transmission. J. Infect. Dis. 199, 889-898.

Kuhn, L., Coutsoudis, A., Moodley, D., Trabattoni, D., Mngqundaniso, N., Shearer, G. M., Clerici, M., Coovadia, H. M., and Stein, Z. (2001). T-helper cell responses to HIV envelope peptides in cord blood: protection against intrapartum and breastfeeding transmission. AIDS 15, 1-9. Legrand, F. A., Nixon, D. F., Loo, C. P., Ono, E., Chapman, J. M., Miyamoto, M., Diaz, R. S., Santos, A. M., Succi, R. C., Abadi, J., Rosenberg, M. G., de Moraes-Pinto, M. I., and Kallas, E. G. (2006). Strong HIV1-specific $\mathrm{T}$ cell responses in HIV1-exposed uninfected infants and neonates revealed after regulatory T cell removal. PLoS ONE 1, e102. doi:10.1371/journal.pone.0000102

Marchant, A., Appay, V., Van Der Sande, M., Dulphy, N., Liesnard, C., Kidd, M., Kaye, S., Ojuola, O., Gillespie, G. M., Vargas Cuero, A. L., Cerundolo, V., Callan, M., McAdam, K. P., Rowland-Jones, S. L., Donner, C., McMichael, A. J., and Whittle, H. (2003). Mature CD8(+) T lymphocyte response to viral infection during fetal life. J. Clin. Invest. 111, 1747-1755.

Miles, D. J., van der Sande, M., Jeffries, D., Kaye, S., Ojuola, O., Sanneh, M., Cox, M., Palmero, M. S., Touray, E. S., Waight, P., RowlandJones, S., Whittle, H., and Marchant, A. (2008). Maintenance of large subpopulations of differentiated CD8 Tcells two years after cytomegalovirus infection in Gambian infants. PLoS ONE 3, e2905. doi:10.1371/journal.pone.0002905

Mold, J. E., Michaelsson, J., Burt, T. D., Muench, M. O., Beckerman, K. P., Busch, M. P., Lee, T. H., Nixon,
D. F., and McCune, J. M. (2008). Maternal alloantigens promote the development of tolerogenic fetal regulatory T cells in utero. Science 322, 1562-1565.

Ogg, G. S., Jin, X., Bonhoeffer, S., Moss, P., Nowak, M. A., Monard, S., Segal, J. P., Cao, Y., RowlandJones, S. L., Hurley, A., Markowitz, M., Ho, D. D., McMichael, A. J., and Nixon, D. F. (1999). Decay kinetics of human immunodeficiency virusspecific effector cytotoxic T lymphocytes after combination antiretroviral therapy. J. Virol. 73, 797-800.

Rowland-Jones, S. L., Nixon, D. F., Aldhous, M. C., Gotch, F., Ariyoshi, K., Hallam, N., Kroll, J. S., Froebel, K., and McMichael, A. (1993). HIVspecific cytotoxic $\mathrm{T}$-cell activity in an HIV-exposed but uninfected infant. Lancet 341, 860-861.

Sabbaj, S., Edwards, B. H., Ghosh, M. K., Semrau, K., Cheelo, S., Thea, D. M., Kuhn, L., Ritter, G. D., Mulligan, M. J., Goepfert, P. A., and Aldrovandi, G. M. (2002). Human immunodeficiency virus-specific CD8(+) T cells in human breast milk. J. Virol. 76, 7365-7373.

Sabbaj, S., Ghosh, M. K., Edwards, B. H., Leeth, R., Decker, W. D. Goepfert, P. A., and Aldrovandi, G. M. (2005). Breast milk-derived antigen-specific CD8+ $\mathrm{T}$ cells: an extralymphoid effector memory cell population in humans. J. Immunol. 174, 2951-2956.

Sharp, E. R., Barbour, J. D., Karlsson, R. K., Jordan, K. A., Sandberg, J. K., Wiznia, A., Rosenberg, M. G., and Nixon, D. F. (2005). Higher frequency of HIV-1-specific $\mathrm{T}$ cell immune responses in African American children vertically infected with HIV-1. J. Infect. Dis. 192, 1772-1780.

Van Sighem, A., Zhang, S., Reiss, P., Gras, L., van der Ende, M., Kroon,
F., Prins, J., and de Wolf, F. (2008) Immunologic, virologic, and clinical consequences of episodes of transient viremia during suppressive combination antiretroviral therapy. J. Acquir. Immune Defic. Syndr. 48, 104-108.

Walter, J., Ghosh, M. K., Kuhn, L., Semrau, K., Sinkala, M., Kankasa, C., Thea, D. M., and Aldrovandi, G. M. (2009). High concentrations of interleukin 15 in breast milk are associated with protection against postnatal HIV transmission. J. Infect. Dis. 200, 1498-1502.

Conflict of Interest Statement: The authors declare that the research was conducted in the absence of any commercial or financial relationships that could be construed as a potential conflict of interest.

Received: 02 November 2011; accepted: 21 December 2011; published online: 11 January 2012.

Citation: Holditch SJ, Eriksson EM, Tarosso LF, Kuebler PJ, Kallas EG, Nielsen EK, Wiznia AA, Rosenberg $M G$ and Nixon DF (2012) Decay kinetics of HIV-1 specific $T$ cell responses in vertically $H I V-1$ exposed seronegative infants. Front. Immun. 2:94. doi: 10.3389/fimmu.2011.00094

This article was submitted to Frontiers in HIV and AIDS, a specialty of Frontiers in Immunology.

Copyright (C) 2012 Holditch, Eriksson, Tarosso, Kuebler, Kallas, Nielsen, Wiznia, Rosenberg and Nixon. This is an open-access article distributed under the terms of the Creative Commons Attribution Non Commercial License, which permits non-commercial use, distribution, and reproduction in other forums, provided the original authors and source are credited. 